\title{
Purple as a tomato: towards high anthocyanin tomatoes
}

\author{
Silvia Gonzali ${ }^{1}$, Andrea Mazzucato ${ }^{2}$ and Pierdomenico Perata ${ }^{1}$ \\ ${ }^{1}$ Plant Lab, Scuola Superiore Sant'Anna, Piazza Martiri della Libertà 33, 56127 Pisa, Italy \\ ${ }^{2}$ Dipartimento di Agrobiologia e Agrochimica, Università degli Studi della Tuscia, 01100 Viterbo, Italy
}

\begin{abstract}
Anthocyanins are naturally occurring pigments ubiquitously present in plants and, as such, part of the human diet. Owing to their biological activity, anthocyanins have beneficial health effects but, unfortunately, are not present in some important crop plants, such as tomatoes. Recently, a 'purple' tomato, highly enriched with anthocyanins, was produced by the ectopic expression of two selected transcription factors from the ornamental flower snapdragon. In addition to being enriched with anthocyanin, these fruits also prolonged the life of cancer-susceptible mice, suggesting that they have additional health-promoting effects.
\end{abstract}

\section{Anthocyanins and the nutritional value of food}

The colour of flowers and fruits is caused by the presence of different kinds of pigment belonging to the phenylpropanoid and terpenoid classes, whose three major groups are chlorophylls, carotenoids and anthocyanins. In some crops, domestication has possibly resulted in selection for agronomic traits that prevail over colour characteristics, whereas in others, an increase or refinement in the pigments naturally present in the edible tissues has occurred. This is likely to apply to tomato (Solanum lycopersicum L.), the fruit of which is rich in several carotenoid pigments, such as lycopene and phytoene [1], but contains only small quantities of some flavonoids; in addition, and somewhat surprisingly, the fruits do not usually produce anthocyanins [2], unlike the fruit of other Solanaceae, such as eggplant (Solanum melongena L.) or pepper (Capsicum spp).

Vegetables, fruits and plant-derived food, such as bread, are fundamental components of the human diet, and the relative content of compounds that are beneficial to human health is an important subject. Among these, considerable importance is given to the presence of some secondary plant metabolites, such as anthocyanins and other flavonoids, which, owing to their health-promoting effects [3-5], add nutritional value to the plant-derived foods that contain them. As a result, in recent years, an increasing number of different approaches have been adopted to stimulate the synthesis of flavonoids in plants [6,7]. Although most of these studies were carried out in a few model species [6], some crops, such as potato and rice, containing suboptimal amounts of these compounds, have also been genetically modified to increase their flavonoid content $[8,9]$. Some such studies focused also on tomatoes,

Corresponding author: Perata, P. (p.perata@sssup.it). as these are among the most important vegetables worldwide. Recently, the expression in tomato of two selected transcription factors (TFs) involved in anthocyanin production in snapdragon (Antirrhinum majus L.) led to high levels of these flavonoids throughout the fruit tissues, which, as a consequence, were purple coloured [10]. This result demonstrates that the anthocyanin biosynthetic pathway can be fully switched on in fruit of this species if activated appropriately.

\section{Why do tomatoes not synthesize anthocyanins naturally?}

Anthocyanins are an important group of naturally occurring polyphenolic compounds derived from the phenylpropanoid biochemical pathway (Box 1) [11,12]. They are members of the flavonoid class of plant secondary metabolites, whose common basal structure consists of two aromatic benzene rings separated by an oxygenated heterocycle, including three carbon atoms [12]. In the glycosylated forms, anthocyanins are soluble pigments that are stored in the acidic vacuole of cells in flowers and fruits (particularly berries), where they are synthesized to attract pollinators and animals for seed dispersal $[11,12]$. They can also be produced in vegetative tissues in response to stressful events, such as high irradiance, against which they can give protection, acting as both a light screen and scavengers for radical species [13]. The presence of anthocyanins in plant-derived food is also important because their intake in the human diet is associated with protection against coronary heart disease and an improvement in sight [4]. They might also prevent cholesterol-induced atherosclerosis, could have anti-inflammatory and anticarcinogenic activities and could aid in the prevention of obesity and diabetes [4]. The biological effects of anthocyanins and flavonoids have been classically ascribed to their antioxidant activity [3]; however, a more recent emerging view is that they might exert modulatory effects, affecting signalling pathways in animal cells [5].

Tomato plants contain a variety of flavonoids in their vegetative tissues, including anthocyanins [14]. In the fruit, they accumulate only small amounts of naringenin chalcone (a flavonoid biosynthetic pathway intermediate) and some flavonols, such as quercetin and kaempferol glycosides, which are concentrated in the peel but do not synthesize anthocyanins [2,14]. 'Black' or 'purple' fruits often described in some heirloom varieties result from mutations affecting chlorophyll breakdown and carotenoid content but are not related to anthocyanin production [14]. By contrast, the production of anthocyanins in the fruit is 
retained by some taxonomically close wild Solanum species, such as Solanum chilense Dunal (Figure 1a) [15]. Interspecific crosses with wild species transferred the ability to produce small quantities of anthocyanins into the peel of cultivated tomatoes. For example, the dominant gene Anthocyanin fruit (Aft), which induces limited pigmentation upon stimulation by high light intensity, was introgressed into domesticated tomato plants by an interspecific cross with $S$. chilense (Figure 1b) [14,15]. Similarly, the gene Aubergine (Abg), which was introgressed from Solanum lycopersicoides Dunal, can induce a strong and variegated pigmentation in the peel of tomatoes $[14,15]$. Furthermore, the recessive gene atroviolacea (atv), derived from the interspecific cross with Solanum cheesmaniae (L. Riley) Fosberg, has been shown to stimulate strong anthocyanin pigmentation in the entire plant, particularly in vegetative tissues (Figure 1c) [14]. Fruits with either $A f t$ and atv alleles (Figure 1d,e) or $A b g$ and atv alleles have been obtained and have generally shown a higher production of anthocyanins in the peel, ranging in total amount from 1 to $4 \mathrm{mg} \mathrm{g}^{-1}$ fresh weight of peel [14].

The flavonoid biosynthetic pathway has been analysed in different $S$. lycopersicum lines [16], including $S$. lycopersicum v. cerasiforme, which is the subspecies thought to be the most probable wild ancestor of domesticated tomatoes [17]. A major constraint found in the flavonoid biosynthetic pathway is the lack of expression of the chalcone isomerase (CHI) gene (Box 1, Figure Ia) in the peel of the fruit, which is probably caused by a mutation in a fruitspecific element of the promoter [16]. This could explain the high level of naringenin chalcone in this tissue of tomatoes, because this is the main substrate of the CHI enzyme (Box 1, Figure Ia). The biosynthetic pathway, by contrast, seems to be constitutively switched off in the flesh of the fruit, where none of the structural genes analysed are expressed [16]. Reintroducing the expression of $\mathrm{CHI}$ in cultivated tomato fruit, by interspecific crosses with wild tomato species [16] or heterologous expression of $\mathrm{CHI}$

\section{Box 1. The anthocyanin biosynthetic pathway}

Anthocyanins are synthesized through a branch of the flavonoid biosynthetic pathway (Figure la) (reviewed in Refs [11,12]). Genes involved in the pathway can be grouped into two different classes: those encoding the enzymes that catalyse the different reaction steps (structural genes) and those regulating the expression of the structural genes (regulatory genes). The enzymatic reactions of flavonoid biosynthesis and most of the corresponding structural genes have been identified and cloned in several species, including cultivated tomato plants, where many of them have been mapped [32]; most of the relative EST (expressed sequence tag) sequences are deposited in GenBank or in the Sol Genomic Network database (http://www.sgn.cornell.edu).

Regulatory genes encode TFs and other regulatory proteins, which interact with the promoter regions of the structural genes, enabling precise temporal and spatial coordination of their transcription and of the resulting production of anthocyanins. Several studies indicate that two main classes of TFs are involved in anthocyanin production: R2R3 MYB-type and bHLH-type TFs [31]. Recent models indicate that these physically interact and, with the participation of another protein with a 'WD40' repeat (WDR), form a MYB-bHLH-WDR (MBW) transcription complex that is directly responsible for the activation of the structural gene transcription (Figure lb) [31].
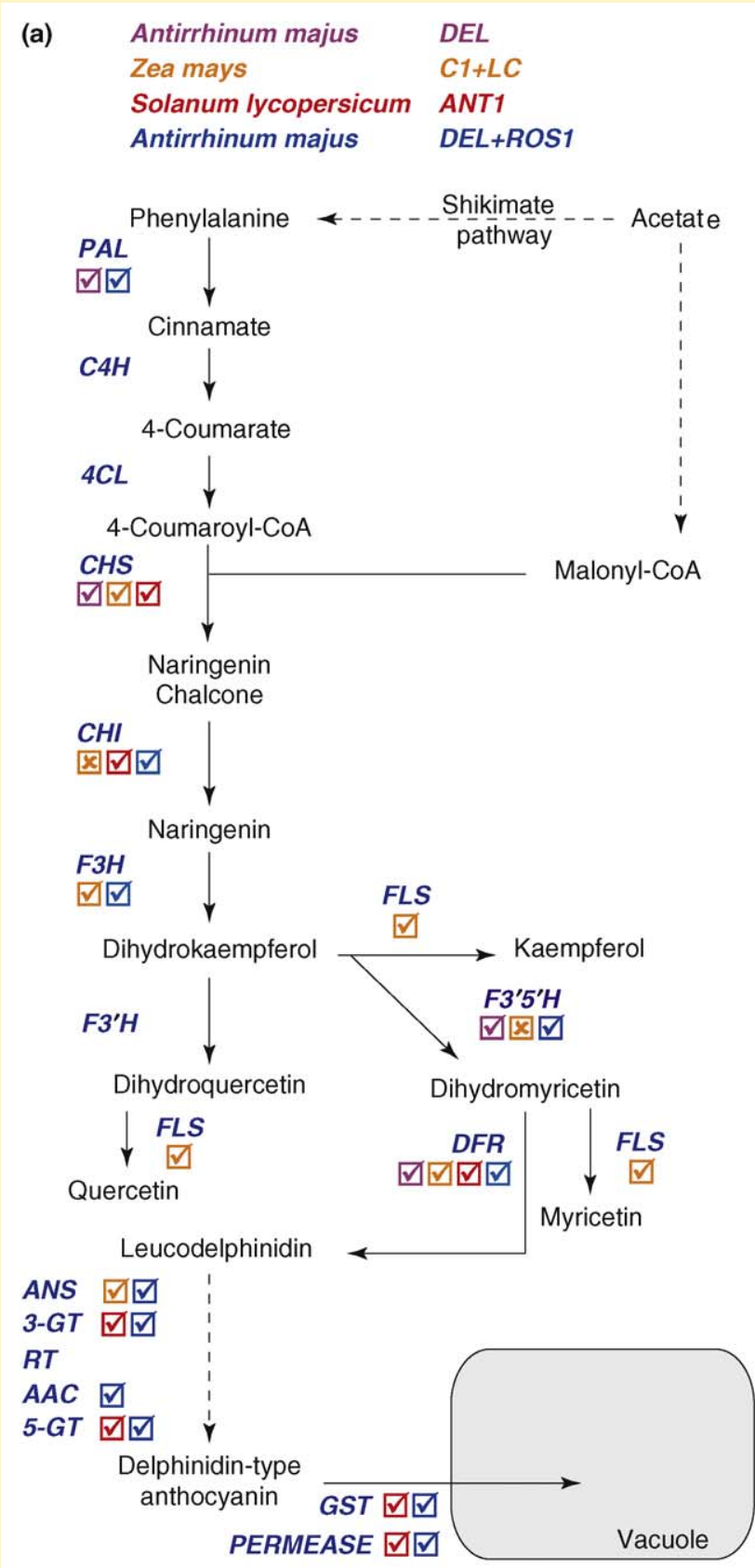

(b)

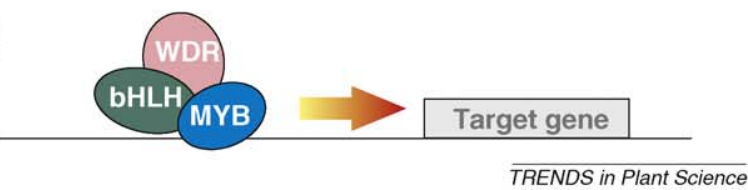

Figure I. Anthocyanin biosynthetic pathway and its regulation. (a) Anthocyanin biosynthesis in tomato plants. Only the production of delphinidin-type anthocyanins, representing the major class observed in tomato plants [23], is shown. Enzyme abbreviations: PAL, phenylalanine ammonia-lyase; $\mathrm{C} 4 \mathrm{H}$, cinnamate-4-hydroxylase; 4CL, 4-coumaroyl:CoA-ligase; CHS, chalcone synthase; $\mathrm{CHI}$, chalcone isomerase; $\mathrm{F} 3 \mathrm{H}$, flavanone 3-hydroxylase; $\mathrm{F}^{\prime} \mathrm{5}^{\prime} \mathrm{H}$, flavonoid $3^{\prime} 5^{\prime}$-hydroxylase; FLS, flavonols synthase; DFR, dihydroflavonol 4reductase; ANS, leucoanthocyanidin dioxygenase; 3-GT, 3-Oglucosyltransferase; RT, rhamnosyl transferase; AAC, anthocyanin acyltransferase; 5-GT, 5-O-glucosyltransferase; GST, glutathione Stransferase; PERMEASE, putative anthocyanin transporter. The TFs that have been expressed in tomato plants are listed at the top of the figure and the corresponding effects on the activation $(\boldsymbol{} \boldsymbol{\omega})$ or lack of effect $(\boldsymbol{X})$ of the main target genes of the pathway (when analysed) are reported, step by step, using coloured symbols. (b) The MBW (MYB-bHLH-WDR) transcription complex responsible for the activation of the structural flavonoid genes [31]. 


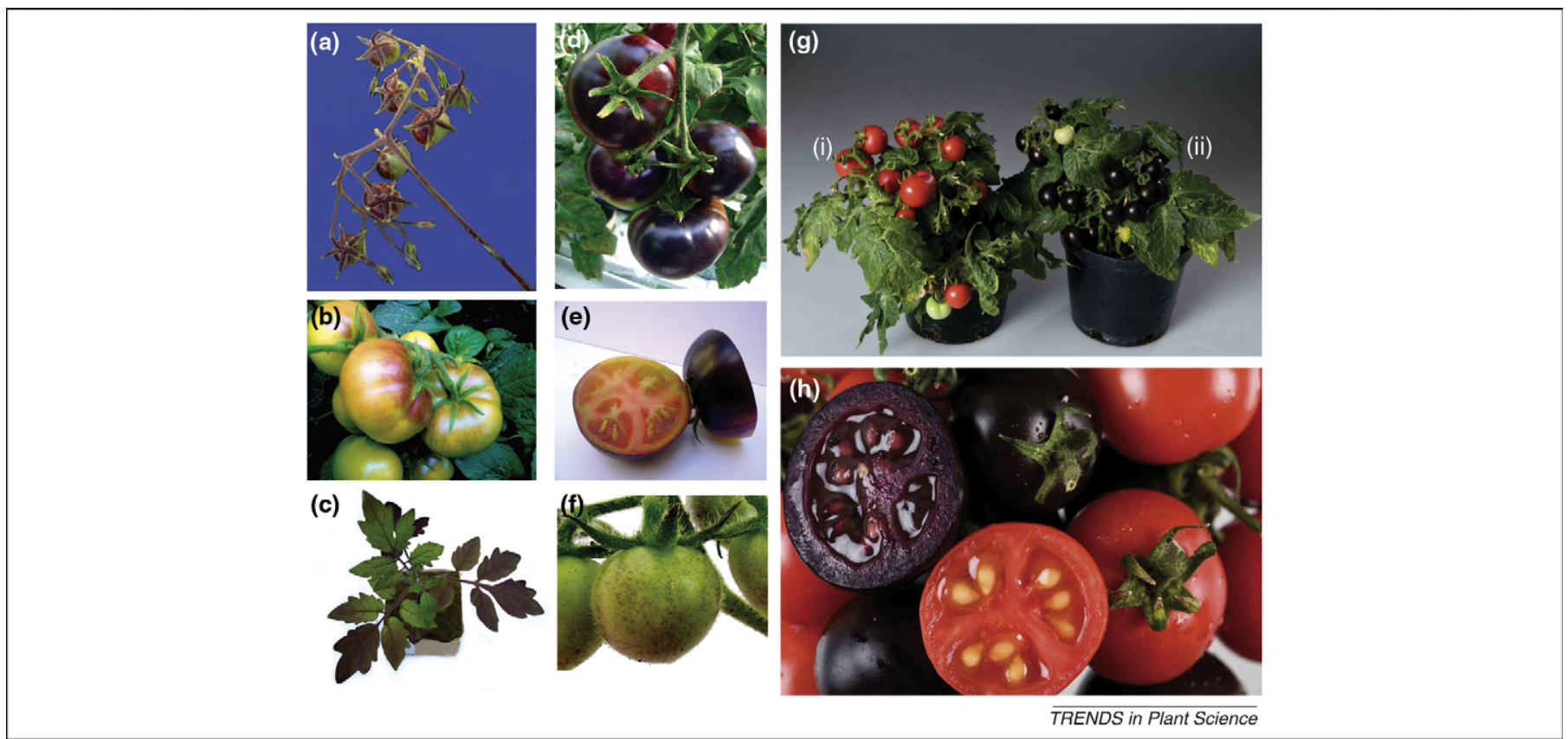

Figure 1. Overview of anthocyanin-enriched tomatoes. (a) Solanum chilense Dunal fruit [15]. (b) Solanum lycopersicum fruit with the Aft gene [15]. (c) S. Iycopersicum plant with the atv gene [14]. (d,e) S. lycopersicum fruit with both the Aft and atv genes [14] (Aft $\times$ atv plants obtained by Gian Piero Soressi). (f) Fruit of S. lycopersicum cv. MicroTom overexpressing AtMYB75 [25]. (g) Comparison of an S. lycopersicum cv. MicroTom control plant (i) with an AmDel/AmRos1-overexpressing line (ii) [10] and (h) sections of the fruit. Reproduced with permission from Roger Chetelat (a); and Andrew Davis and Sue Bunnewell (g,h).

isolated from other species, for example from Petunia [18], results in tomato plants with higher levels of quercetin glycosides in their fruit peel. This shows that, when removing the bottleneck of CHI in the biosynthetic pathway, flavonoids can be synthesized. It is therefore likely that the default state for the peel of tomatoes is to produce these phenolic compounds, as hypothesized [16].

It was also suggested that cultivated tomato might have lost $C H I$ expression in the fruit peel relatively early during domestication or even before, given that this trait was observed in all the cultivars of domesticated tomato analysed, including S. lycopersicum v. cerasiforme [16]. It is tempting to speculate that, because the fruit peel of some wild tomato species and of other Solanaceae (e.g. eggplant [19] and pepper [20]) can accumulate anthocyanins, other natural mutations occurred in regulatory and/or structural genes of the flavonoid biosynthetic pathway, affecting not only flavonol production but also anthocyanin synthesis in tomatoes [21]. Certainly, further studies are necessary to clarify this point.

\section{Engineering anthocyanin accumulation in tomato fruit: towards a 'purple' tomato}

Recently, various transgenic approaches have been taken to increase flavonoid levels in tomato fruit by overexpressing either the structural or regulatory genes involved in the biosynthetic pathway (Box 1). Most of these attempts have exploited heterologous genes [18,22-26], and in only one case was an endogenous tomato regulatory gene (ANT1, encoding a MYB-type TF [Box 1]) overexpressed in tomato plants [27]. Although a significant increase in the final content of some flavonoids (flavonols in particular) was obtained in several cases [18,23,24,26], only partial success was achieved in relation to the production of anthocyanins. For example, the overexpression of the basic helix-loop-helix (bHLH) TF (Box 1) Delila (Del) of snapdragon induced strong pigmentation only in the vegetative tissues of the plant [22], whereas the overexpression of both tomato ANT1 [27] and Arabidopsis PRODUCTION OF ANTHOCYANIN PIGMENT1 (PAP1, also called MYB75) (Figure 1f) [25] MYB-type TFs induced only a partial, spotted pigmentation, mostly limited to the peel of the fruit. Whereas the lack of activation of some structural genes was the major cause when no anthocyanins were produced (Box 1, Figure Ia), in the case of partial pigmentation, the low availability of flavonoid biosynthetic precursors or the absence of other limiting regulatory factors (e.g. bHLH TFs) could explain the failure to activate full pigmentation.

Alternative approaches to improving the nutritional value of tomato fruit have also been taken. In particular, manipulation of genes encoding components of the lightsignalling transduction machinery can affect the final content of secondary metabolites, as shown by the fruitspecific RNA interference (RNAi)-mediated suppression of DE-ETIOLATED1 (DET1), a photomorphogenesis regulating gene. Inactivation of $D E T 1$ led to a significant increase in both flavonoids and carotenoids in tomato fruit [28].

These constraints encountered when trying to obtain high-anthocyanin tomato fruits were overcome in the most recent attempt to enrich tomato fruit with anthocyanins, by Eugenio Butelli et al. [10]. These authors selected two different snapdragon TFs - Del, which is a bHLH-type TF, and Rosea1 (Ros1), an R2R3 MYB-type TF - and overexpressed them in tomato (cv. MicroTom) under the control of a fruit-specific E8 promoter. Whereas transgenic plants showed normal vegetative growth, transgenic fruit started to synthesize anthocyanins at the end of the green stage and continued to accumulate these pigments during subsequent ripening, ultimately reaching an intense, uniform 
purple colouration both in the peel and in the flesh (Figure $1 \mathrm{~g}, \mathrm{~h}$ ). In the fruit of the line with the strongest visible pigmentation, the final concentration of anthocyanins averaged $\sim 3 \mathrm{mg} \mathrm{g}^{-1}$ fresh weight [10], which is the highest value obtained so far in this species.

This striking phenotype was due to a combination of factors. First, Del and Ros1 stimulated the transcription of most of the structural genes involved in the biosynthetic pathway, including phenylalanine ammonia-lyase (PAL), $C H I$ and flavonoid $3^{\prime} 5^{\prime}$-hydroxylase $\left(F 3^{\prime} 5^{\prime} H\right)$ [10]. PAL induction is necessary to ensure high levels of flux through the general phenylpropanoid metabolism to feed flavonoid biosynthesis [29], whereas $C H I$ and $F 3^{\prime} 5^{\prime} H$ are both essential to address the flux of flavonoid intermediates towards the anthocyanin products (Box 1, Figure Ia). $P A L, C H I$ and $F 3^{\prime} 5^{\prime} H$ were not induced when other heterologous anthocyanin-related $\mathrm{TF}$ genes, such as maize (Zea mays L.) Leaf colour $(L C)$ and Colourless1 (C1), were expressed in tomato plants, resulting in a higher levels of flavonols, but no anthocyanins [23]. Such a difference confirms that $F 3^{\prime} 5^{\prime} H$ expression is necessary to activate anthocyanin synthesis in tomatoes [23] because only delphinidin-type anthocyanins, which derive from dihydromyricetin and therefore require $\mathrm{F}^{\prime}{ }^{\prime}{ }^{\prime} \mathrm{H}$ enzymatic activity (Box 1, Figure Ia), are produced in these plants. At the same time, stronger activation of the entire phenylpropanoid pathway, caused by the significant increase in both $P A L$ transcription and PAL enzymatic activity and in the expression of $C H I$, seems to be important to obtain very high levels of anthocyanins, such as those observed in Del-Ros1 transgenic plants [10].

Second, Del and Ros1 also stimulated genes involved in the side-chain modification of the anthocyanin pigments and genes possibly related to the final transport of these molecules into the vacuole [10], processes that are both necessary for the accumulation of anthocyanin (Box 1, Figure Ia). Therefore, although TFs from different plant species are involved in the same biosynthetic process, they are characterized by a different specificity in their target genes, which might be particularly marked in heterologous host species [30]. A sustained expression of both bHLH and MYB TFs (Box 1, Figure Ib) is also necessary to obtain a uniform and widespread synthesis of pigments within the fruit.

The high production of anthocyanins in these 'purple' tomatoes was not obtained at the expense of other major classes of tomato fruit pigments, and the antioxidant activity attributable to the carotenoid fraction remained unchanged [10]. This could be due to the careful selection of the promoter (E8) for expression of regulatory genes. Expression late in fruit development meant that anthocyanin biosynthesis did not interfere with other metabolic processes occurring earlier in fruit ripening. These tomatoes therefore fulfil the requirements in terms of total (lipophilic [from carotenoids] + hydrophilic [from flavonoids]) antioxidant activity.

Importantly, in a pilot experiment, a group of cancersusceptible mice that were fed a diet supplemented with these high-anthocyanin tomatoes showed a significant extension of their average life span compared with cancer-susceptible mice that were fed a diet supplemented with regular control tomatoes [10]. This demonstrates how an anthocyanin-enriched tomatoderived food can be beneficial for animal health. Further studies are clearly required to confirm the potential beneficial role of these anthocyanin-rich tomatoes in human health.

\section{Perspectives}

The work of Butelli et al. [10] and previous related papers highlight the interest in the production of crop plants enriched with health-promoting compounds. Among these, anthocyanins and flavonoids are some of the most important targets for improving the nutritional value of foodstuffs. Extensive knowledge of the molecular mechanisms underlying the elaborate and sophisticated biosynthetic pathways of secondary metabolites in plants is essential to drive metabolic engineering strategies in the most rewarding direction, as Butelli et al. [10] have shown.

Furthermore, given that the biological properties of different anthocyanin compounds and of the other classes of flavonoids are often molecule-specific, a major objective of metabolic engineering approaches might be not only increasing but also optimizing their levels and composition in crops. This could be done, for example, by combining the use of regulatory and structural genes. Additionally, researchers could exploit natural genetic resources, either as an alternative to genetic engineering approaches or to strengthen them. The identification of the complete set of endogenous regulatory proteins involved in flavonoid metabolism in tomato and its close wild species, in particular the characterization of the mutations $A f t, A b g$ and atv, will thus contribute in this sense to the production of high nutritional value tomatoes. Further work is also needed to investigate the potential health benefits of these unusual, but colourful, tomatoes.

\section{Acknowledgements}

We thank Eugenio Butelli for helpful comments on a draft of this paper and for providing the photographs shown in Figure $1 \mathrm{~g}, \mathrm{~h}$; Gian Piero Soressi for providing the seeds used to produce the plants shown in Figure 1d,e; and Roger Chetelat for providing the photograph of $S$. chilense (Figure 1a), taken by Carl Jones. This research was supported by the Italian Ministry of University and Research (MiUR), PRIN2006, TomANTHO Project.

\section{References}

1 Khachik, F. et al. (2002) Chemistry, distribution, and metabolism of tomato carotenoids and their impact on human health. Exp. Biol. Med. (Maywood) 227, 845-851

2 Torres, C.A. et al. (2005) Disposition of selected flavonoids in fruit tissues of various tomato (Lycopersicon esculentum Mill.) genotypes. J. Agric. Food Chem 53, 9536-9543

3 Pietta, P-G. (2000) Flavonoids as antioxidants. J. Nat. Prod. 63, 10351042

4 Lila, M.A. (2004) Anthocyanins and human health: an in vitro investigative approach. J. Biomed. Biotechnol. 2004, 306-313

5 Williams, R.J. et al. (2004) Flavonoids: antioxidant or signalling molecules? Free Radic. Biol. Med. 36, 838-849

6 Schijlen, E.G. et al. (2004) Modification of flavonoid biosynthesis in crop plants. Phytochemistry 65, 2631-2648

7 Tanaka, Y. and Ohmiya, A. (2008) Seeing is believing: engineering anthocyanin and carotenoid biosynthetic pathways. Curr. Opin. Biotechnol. 19, 190-197

8 Lukaszewicz, M. et al. (2004) Antioxidant capacity manipulation in transgenic potato tuber by changes in phenolic compounds content. $J$. Agric. Food Chem. 52, 1526-1533 
9 Reddy, A.M. et al. (2007) Novel transgenic rice overexpressing anthocyanidin synthase accumulates a mixture of flavonoids leading to an increased antioxidant potential. Metab. Eng. 9, 95-111

10 Butelli, E. et al. (2008) Enrichment of tomato fruit with healthpromoting anthocyanins by expression of select transcription factors. Nat. Biotechnol. 26, 1301-1308

11 Koes, R. et al. (2005) Flavonoids: a colorful model for the regulation and evolution of biochemical pathways. Trends Plant Sci. 10, 236242

12 Tanaka, Y. et al. (2008) Biosynthesis of plant pigments: anthocyanins, betalains and carotenoids. Plant J. 54, 733-749

13 Gould, K.S. (2004) Nature's Swiss army knife: the diverse protective roles of anthocyanins in leaves. J. Biomed. Biotechnol. 2004, 314320

14 Mes, P.J. et al. (2008) Characterization of tomatoes expressing anthocyanin in the fruit. J. Am. Soc. Hortic. Sci. 133, 262-269

15 Jones, C.M. et al. (2003) Characterization and inheritance of the Anthocyanin fruit (Aft) tomato. J. Hered. 94, 449-456

16 Willits, M.G. et al. (2005) Utilization of the genetic resources of wild species to create a nontransgenic high flavonoid tomato. J. Agric. Food Chem. 53, 1231-1236

17 Nesbitt, T.C. and Tanksley, S.D. (2002) Comparative sequencing in the genus Lycopersicon: implications for the evolution of fruit size in the domestication of cultivated tomatoes. Genetics 162, 365-379

18 Muir, S.R. et al. (2001) Overexpression of petunia chalcone isomerase in tomato results in fruit containing dramatically increased levels of flavonols. Nat. Biotechnol. 19, 470-474

19 Azuma, K. et al. (2008) Structures and antioxidant activity of anthocyanins in many accessions of eggplant and its related species. J. Agric. Food Chem. 56, 10154-10159

20 Lightbourn, G.J. et al. (2008) Effects of anthocyanin and carotenoid combinations on foliage and immature fruit color of Capsicum annuum L. J. Hered. 99, 105-111

21 Doganlar, S. et al. (2002) Conservation of gene function in the Solanaceae as revealed by comparative mapping of domestication traits in eggplant. Genetics 161, 1713-1726
22 Mooney, M. et al. (1995) Altered regulation of tomato and tobacco pigmentation genes caused by the Delila gene of Antirrhinum. Plant J. 7, 333-339

23 Bovy, A. et al. (2002) High-flavonol tomatoes resulting from the heterologous expression of the maize transcription factor genes $L C$ and C1. Plant Cell 14, 2509-2526

24 Verhoeyen, M.E. et al. (2002) Increasing antioxidant levels in tomatoes through modification of the flavonoid biosynthetic pathway. J. Exp. Bot. 53, 2099-2106

25 Zuluaga, D.L. et al. (2008) Arabidopsis thaliana MYB75/PAP1 transcription factor induces anthocyanin production in transgenic tomato plants. Funct. Plant Biol. 35, 606-618

26 Luo, J. et al. (2008) AtMYB12 regulates caffeoyl quinic acid and flavonol synthesis in tomato: expression in fruit results in very high levels of both types of polyphenol. Plant J. 56, 316-326

27 Mathews, H. et al. (2003) Activation tagging in tomato identifies a transcriptional regulator of anthocyanin biosynthesis, modification, and transport. Plant Cell 15, 1689-1703

28 Davuluri, G.R. et al. (2005) Fruit-specific RNAi-mediated suppression of DET1 enhances carotenoid and flavonoid content in tomatoes. Nat. Biotechnol. 23, 890-895

29 Bate, N.J. et al. (1994) Quantitative relationship between phenylalanine ammonia-lyase levels and phenylpropanoid accumulation in transgenic tobacco identifies a rate-determining step in natural product synthesis. Proc. Natl. Acad. Sci. U. S. A. 91, $7608-7612$

30 Schwinn, K. et al. (2006) A small family of MYB-regulatory genes controls floral pigmentation intensity and patterning in the genus Antirrhinum. Plant Cell 18, 831-851

31 Quattrocchio, F. et al. (2006) The regulation of flavonoid biosynthesis. In The Science of Flavonoids (Grotewold, E., ed.), pp. 97-122, Springer

32 De Jong, W.S. (2004) Candidate gene analysis of anthocyanin pigmentation loci in the Solanaceae. Theor. Appl. Genet. 108, 423-432

1360-1385/\$ - see front matter ๑ 2009 Elsevier Ltd. All rights reserved. doi:10.1016/j.tplants.2009.02.001 Available online 8 April 2009 